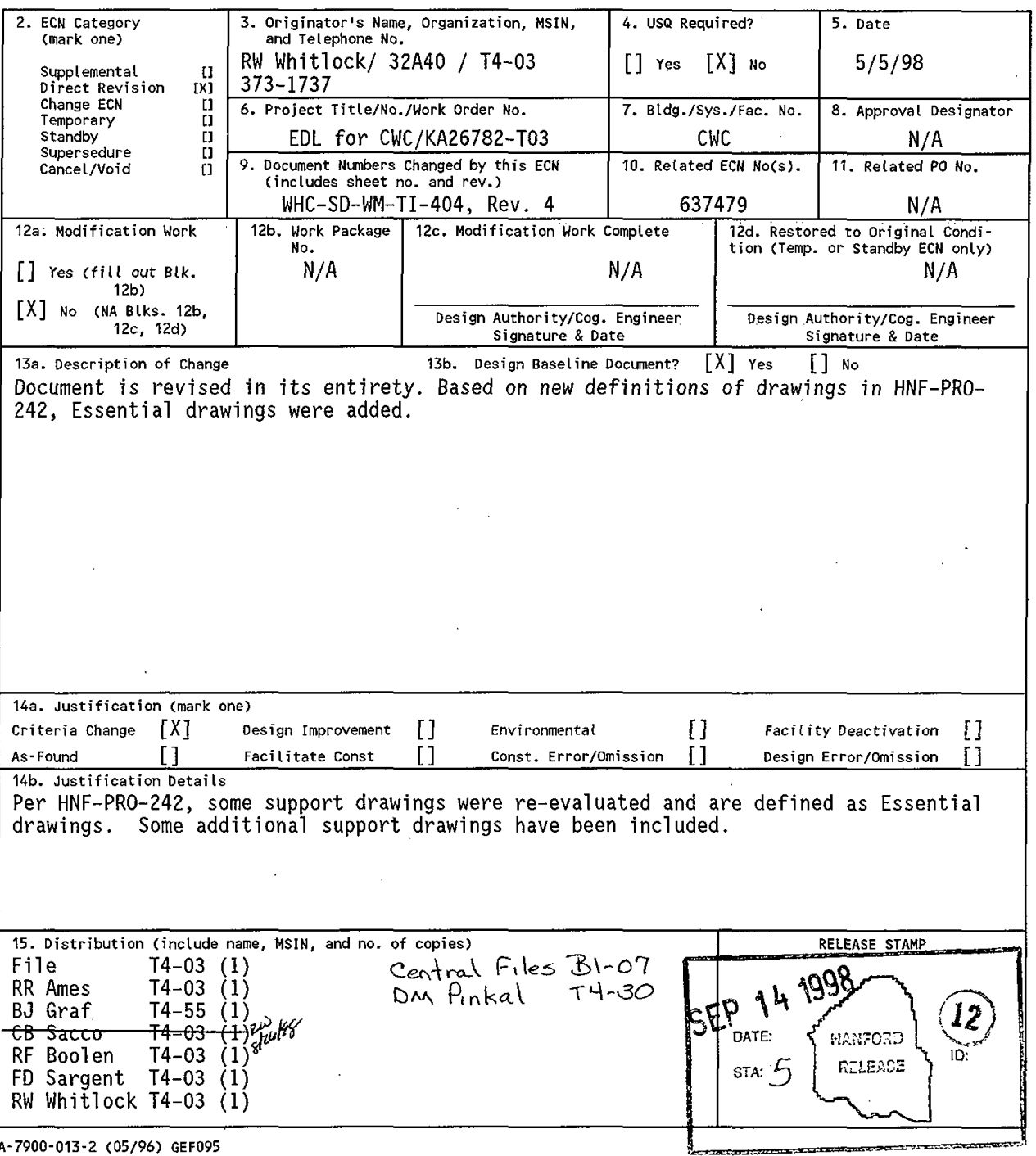




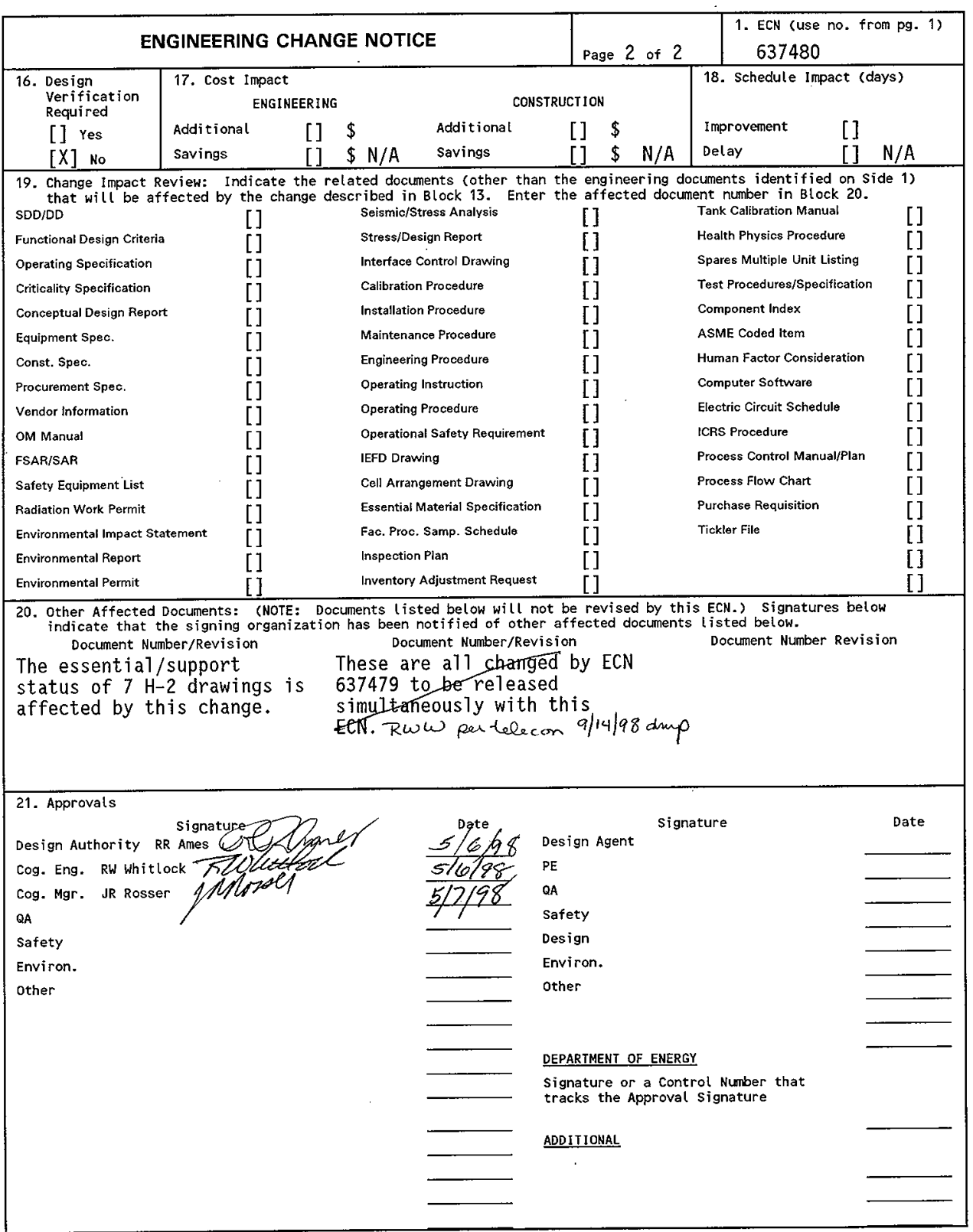




\section{Central Waste Complex (CWC) Essential/Support Drawing List}

R. W. Whitlock

Waste Management Eederal Services of Hanford, Inc.

Richland, WA 99352

U.S. Department of Energy Contract DE-AC06-96RL13200

\begin{tabular}{|c|c|c|c|}
\hline EDT/ECN: & $E C N-637480$ & UC: 510 & \\
\hline & $32 \AA 40$ & Charge Code: & $A 1 E \perp B$ \\
\hline B\&R Code & EW3130020 & Total Pages: & 10 \\
\hline
\end{tabular}

Key Words: Drawings, essential, support, Central Waste Complex

Abstract: This document lists the essential and support drawings for the Central waste Complex

TRADEMARK DISCLAIMER. Reference herein to any specific commercial product, process, or service by trade name, trademark, manufacturer, or otherwise, does not necessarily constitute or imply its endorsement, recommendation, or favoring by the United States Government or any agency thereof or its contractors or subcontractors.

Printed in the United States of America. To obtain copies of this document, contact: Document Control Services, P.O. Box 950, Mailstop H6-08, Richland WA 99352, Phone (509) 372-2420; Fax (509) 376-4989.
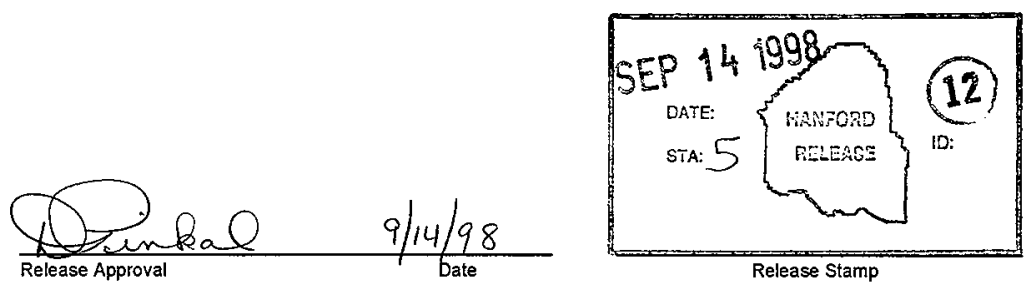

\section{Approved For Public Release}


(2) Title

Central Waste Complex (CWC) Essential/Support Drawing List

CHANGE CONTROL RECORD

\begin{tabular}{|c|c|c|c|c|}
\hline \multirow{2}{*}{ (3) } & \multirow{2}{*}{ Revision } & \multirow{2}{*}{ (4) Description of Change - Replace, Add, and Delete Pages } & \multicolumn{2}{|c|}{ Authorized for Release } \\
\hline & & & (5) Cog. Engr. & (6) Cog. Mgr. \\
\hline & 0 & (7) Original EDT-151759 & & \\
\hline & 1 & $\begin{array}{l}\text { This document is an update to the "Central } \\
\text { Waste Complex EssentiaT/Support Drawing } \\
\text { List" WHC-SD-WM-TI-404 Rev. 0. Changes are } \\
\text { per ECN } 157598\end{array}$ & & \\
\hline & 2 & $\begin{array}{l}\text { Added the } 2403 \text { series Building drawings to } \\
\text { the 1ist. ECN } 600780\end{array}$ & PMR 9/27/93 & $\begin{array}{l}\text { JA James for DB } \\
\text { Powe11 } 9 / 27 / 93\end{array}$ \\
\hline & 3 & $\begin{array}{l}\text { - Complete the revision of this supporting } \\
\text { document per ECN } 615378 \text { (Added fire } \\
\text { protection drawing sand added drawings from } \\
213-W \text { ) } \\
\text { - Supersedes WHC-SD-WM-TI-400 per ECN } \\
615380 \\
\text { - Incorporates changes from ECN's: } 605631 \text {, } \\
608522 \text {, } 602648,615376,615379,157600, \& \\
153871\end{array}$ & LJ Gaschott & DS Powell \\
\hline & 4 & $\begin{array}{ll}\text { Deletes } 213-W & \text { drawings from list. } \\
\text { evaluates all remaining drawings. } & \text { ECN } \\
623645 & \\
\end{array}$ & KM McDonald & $\begin{array}{l}\text { DB Powe11 dated } \\
8 / 31 / 95\end{array}$ \\
\hline RS & 5 & $\begin{array}{l}\text { Re-evaluates al1 drawings per HNF-PR0-242. } \\
\text { Incorporates the following ECN's: } 637479 \text {, } \\
626121,633695,620874 \text {. }\end{array}$ & RW Whitlogk & $\begin{array}{l}\text { JR Rosser } \\
\text { Horgh } 9 / 6 / 90\end{array}$ \\
\hline & & & 98 dimp & \\
\hline & & & & \\
\hline & & & & \\
\hline & & & & \\
\hline & & & & \\
\hline & & & & \\
\hline & & & & \\
\hline & & & & \\
\hline & & & & \\
\hline & & 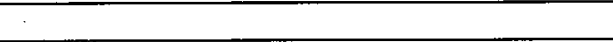 & & \\
\hline & & & & \\
\hline & & & & \\
\hline & & & & \\
\hline & & & & \\
\hline & & & & \\
\hline & & & & \\
\hline
\end{tabular}




\section{CENTRAL WASTE COMPLEX (CWC) ESSENTIAL/SUPPORT DRAWING LIST}

\subsection{INTRODUCTION}

Essential and supporting engineering drawings for the Central Waste Complex (CWC) are identified in this document. The purpose of the document is to describe the criteria used to identify drawings and the plan for updating and maintaining their accuracy. This document supports HNF-PRO-242 and HNF-PRO-440.

\subsection{SELECTION PROCESSES}

Essential and support drawings are identified by the facility cognizant engineer and concurred with by the cognizant engineering manager. The selection criteria are presented below (see PRO-242, Standard Engineering Practices, Giossary).

\section{ESSENTIAL DRAWINGS}

Those engineering drawings identified by the facility staff as necessary to directly support the safe operation or maintenance of the facility.

\section{SUPPORT DRAWINGS}

Those engineering drawings identified by the facility staff that further describe the design details of structures, systems, or components shown on essential drawings or are frequently used by the support staff.

\section{GENERAL DRAWINGS}

Those engineering drawings identified by the facility staff as historical in nature, or not frequently used in the operation or maintenance of the facility, and that are not identified as either essential or support drawings.

A Tisting of essential drawings are in Table 1. A listing of support drawings are provided in Table 2. Other drawings associated with the facility are designated as general drawings.

\subsection{MAINTENANCE OF DRAWINGS}

Copies of all essential and support drawings will be available to the cognizant engineer at his or her work location. An as-built file of all drawings will be maintained by the engineering organization. 
HNF-SD-WM-TI-404, Rev. 5

Table 1. Essential Drawings.

\begin{tabular}{||c|c|c|l||}
\hline $\begin{array}{c}\text { Drawing } \\
\text { No. }\end{array}$ & Sheet & Building & \multicolumn{1}{|c|}{ Title } \\
\hline $\mathrm{H}-2-80544$ & 1 & $2401 \mathrm{~W}$ & Elec/Fire Alm Plan Dets \& Scheds \\
\hline $\mathrm{H}-2-80743$ & 1 & $2402 \mathrm{~W}$ & Electrical Plans Diagram \& Detai1s \\
\hline $\mathrm{H}-2-80743$ & 2 & $2402 \mathrm{~W}$ & Electrical Plans, Diagram and Detai1s \\
\hline $\mathrm{H}-2-80743$ & 3 & $2402 \mathrm{~W}$ & Electrical Plans, Diagram and Detai1s \\
\hline $\mathrm{H}-2-131547$ & 1 & $2403 \mathrm{WA}$ & Electrical Plan \& Diagrams \\
\hline $\mathrm{H}-2-131547$ & 2 & $2403 \mathrm{~W}$ & Electrical Plan \& Diagrams \\
\hline $\mathrm{H}-2-80923$ & 1 & $2403 \mathrm{WD}$ & Elec Diag \& Pnlbd Sched \\
\hline $\mathrm{H}-2-80924$ & 1 & $2403 \mathrm{WB}$ & Elec Diag \& Pnlbd Sched \\
\hline $\mathrm{H}-2-80925$ & 1 & $2403 \mathrm{WC}$ & Elec Diag \& Pnlbd Sched \\
\hline $\mathrm{H}-2-823233$ & 1 & $2404 \mathrm{~W}$ & Electrical Floor Plans \& Notes \\
\hline $\mathrm{H}-2-823233$ & 2 & $2404 \mathrm{~W}$ & Electrical Floor Plans \& Notes \\
\hline $\mathrm{H}-2-823234$ & 1 & $2404 \mathrm{~W}$ & Electrical One-Line Panels \& Detai7s \\
\hline $\mathrm{H}-2-72500$ & 1 & $2727 \mathrm{~W}$ & Wiring Dia. Fire Protection \\
\hline
\end{tabular}


HNF-SD-WM-TI-404, Rev. 5

Table 2. Support Drawings.

\begin{tabular}{|c|c|c|c|}
\hline $\begin{array}{l}\text { Drawing } \\
\text { No. }\end{array}$ & Sheet & Building & Title \\
\hline $\mathrm{H}-2-80544$ & 2 & $2401 W$ & Elec/Fire Alm Plan Dets \& Scheds \\
\hline $\mathrm{H}-2-80545$ & 1 & $2401 W$ & Arch/Strl Elev \& Dets \\
\hline $\mathrm{H}-2-80545$ & 2 & $2401 W$ & Arch/Str] Elevations \& Details \\
\hline $\mathrm{H}-2-80606$ & 1 & $2402 W$ & Arch/Strl Sections \& Details \\
\hline $\mathrm{H}-2-80606$ & 2 & $2402 W$ & Arch/Strl Sections \& Details \\
\hline $\mathrm{H}-2-80607$ & 1 & $2402 \mathrm{~W}$ & Electrical Plan, Section, Details \\
\hline $\mathrm{H}-2-80607$ & 2 & $2402 W$ & Electrical Plan, Section, Details \\
\hline$H-2-80608$ & 1 & $2402 W$ & $\begin{array}{l}\text { Fire Protection Fire Alarm Plans El } \\
\text { Det \& Diag }\end{array}$ \\
\hline $\mathrm{H}-2-80609$ & 1 & $2402 \mathrm{~W}$ & Elec Site Plan \& Pole Line Dets \\
\hline$H-2-80739$ & 1 & $2402 W$ & $\begin{array}{l}\text { Arch/Strl Plan, Elevations, Section, } \\
\text { Details }\end{array}$ \\
\hline$H-2-80740$ & 1 & $2402 W$ & $\begin{array}{l}\text { Fire Protection Fire Alarm Sys Plans } \\
\& \text { Se }\end{array}$ \\
\hline $\mathrm{H}-2-80740$ & 2 & $2402 \mathrm{~W}$ & $\begin{array}{l}\text { Fire Protection Alarm Sys Plans Sect } \\
\text { Det }\end{array}$ \\
\hline$H-2-80741$ & 1 & $2402 W$ & $\begin{array}{l}\text { Fire Protection Fire Alarm Sys Wiring } \\
\text { Dia }\end{array}$ \\
\hline $\mathrm{H}-2-80741$ & 2 & $2402 W$ & Fire Protection ATarm Sys Wiring Diag \\
\hline $\mathrm{H}-2-80742$ & 1 & $2402 W$ & Elec Site PTan Symbol List \& ScheduTe \\
\hline $\mathrm{H}-2-821880$ & 1 & $2402 W$ & Fire Protection Sprinkler System \\
\hline $\mathrm{H}-2-821880$ & 2 & $2402 W$ & $\begin{array}{l}\text { Fire Protection System Details and } \\
\text { Sections }\end{array}$ \\
\hline$H-2-821881$ & 1 & $2402 \mathrm{WB}$ & Fire Protection Sprinkler system \\
\hline $\mathrm{H}-2-821881$ & 2 & $2402 W B$ & $\begin{array}{l}\text { Fire Protection Sprinkler System } \\
\text { Detail and Sect }\end{array}$ \\
\hline $\mathrm{H}-2-821882$ & 1 & $2402 W C$ & Fire Protection Sprinkler System \\
\hline $\mathrm{H}-2-821882$ & 2 & $2402 W C$ & $\begin{array}{l}\text { Fire Protection Sprinkler system } \\
\text { Details and Sections }\end{array}$ \\
\hline $\mathrm{H}-2-821883$ & 1 & $2402 W D$ & Fire Protection Sprinkler System \\
\hline $\mathrm{H}-2-821883$ & 2 & $2402 W D$ & $\begin{array}{l}\text { Fire Protection Sprinkler System } \\
\text { Details and Sections }\end{array}$ \\
\hline $\mathrm{H}-2-821884$ & 1 & 2402WE & Fire Protection Sprinkler System \\
\hline
\end{tabular}


HNF-SD-WM-TI-404, Rev. 5

Tabie 2. Support Drawings (cont.).

\begin{tabular}{|c|c|c|c|}
\hline $\begin{array}{l}\text { Drawing } \\
\text { No. }\end{array}$ & Sheet & Building & Title \\
\hline $\mathrm{H}-2-821884$ & 2 & 2402WE & $\begin{array}{l}\text { Fire Protection Sprinkler System } \\
\text { Details and Sections }\end{array}$ \\
\hline $\mathrm{H}-2-821885$ & 1 & $2402 \mathrm{WF}$ & Fire Protection Sprinkler System \\
\hline$H-2-821885$ & 2 & $2402 W F$ & $\begin{array}{l}\text { Fire Protection Sprinkler System } \\
\text { Details and Sections }\end{array}$ \\
\hline $\mathrm{H}-2-821886$ & 1 & $2402 W G$ & Fire Protection Sprinkler System \\
\hline $\mathrm{H}-2-821886$ & 2 & $2402 W G$ & $\begin{array}{l}\text { Fire Protection Sprinkler System } \\
\text { Details and Sections }\end{array}$ \\
\hline $\mathrm{H}-2-821887$ & 1 & $2402 W H$ & Fire Protection Sprinkler System \\
\hline H-2-821887 & 2 & $2402 W H$ & $\begin{array}{l}\text { Fire Protection Sprinkler System } \\
\text { Details and Sections }\end{array}$ \\
\hline $\mathrm{H}-2-821888$ & 1. & $2402 W I$ & Fire Protection Sprinkler System \\
\hline$H-2-821888$ & 2 & $2402 W I$ & $\begin{array}{l}\text { Fire Protection Sprinkler System } \\
\text { Details and Sections }\end{array}$ \\
\hline $\mathrm{H}-2-821889$ & 1 & $2402 \mathrm{WJ}$ & Fire Protection Sprink]er System \\
\hline $\mathrm{H}-2-821889$ & 2 & $2402 W J$ & $\begin{array}{l}\text { Fire Protection Sprinkler System } \\
\text { Details and Sections }\end{array}$ \\
\hline $\mathrm{H}-2-821890$ & 1 & $2402 W K$ & Fire Protection Sprinkler System \\
\hline$H-2-821890$ & 2 & $2402 \mathrm{WK}$ & $\begin{array}{l}\text { Fire Protection Sprinkler System } \\
\text { Details and Sections }\end{array}$ \\
\hline $\mathrm{H}-2-821891$ & 1 & $2402 \mathrm{WL}$ & Fire Protection Sprinkler System \\
\hline$H-2-821891$ & 2 & $2402 W L$ & $\begin{array}{l}\text { Fire Protection Sprinkler System } \\
\text { Details and Sections }\end{array}$ \\
\hline $\mathrm{H}-2-131542$ & 1 & $2403 W A$ & Fire Protection Plan Sect \& Details \\
\hline $\mathrm{H}-2-131543$ & 1 & 2403WA & $\begin{array}{l}\text { Fire Protection Alarm System Wiring } \\
\text { Diag }\end{array}$ \\
\hline $\mathrm{H}-2-131545$ & 1 & 2403WA & Instrumentation CAM Enclosure \\
\hline $\mathrm{H}-2-131545$ & 2 & 2403WA & Instrumentation CAM Enclosure \\
\hline H-2-131546 & 1 & 2403WA & $\begin{array}{l}\text { Instr Cam Alarm Intcon Diag \& Instr } \\
\text { Encl. }\end{array}$ \\
\hline $\mathrm{H}-2-131546$ & 2 & $2403 \mathrm{WA}$ & Instr Cam Alarm Elem Diag \\
\hline $\mathrm{H}-2-131546$ & 3 & $2403 \mathrm{WA}$ & Air Sampler Alarm Elementary Diagram \\
\hline $\mathrm{H}-2-131548$ & 1 & 2403WA & Electrical Elevations \& Details \\
\hline
\end{tabular}


HNF-SD-WM-TI-404, Rev. 5

Table 2. Support Drawings (cont.).

\begin{tabular}{|c|c|c|c|}
\hline $\begin{array}{l}\text { Drawing } \\
\text { No. }\end{array}$ & Sheet & Building & Title \\
\hline $\mathrm{H}-2-131548$ & 2 & 2403WA & Electrical Details \\
\hline $\mathrm{H}-2-821893$ & 1 & $2403 W A$ & Fire Protection Sprinkler System \\
\hline$H-2-821893$ & 2 & 2403WA & $\begin{array}{l}\text { Fire Protection Sprinkler System } \\
\text { Details and Sec }\end{array}$ \\
\hline$H-2-821893$ & 3 & 2403WA & $\begin{array}{l}\text { Fire Protection Sprinkler System } \\
\text { Details and Sec }\end{array}$ \\
\hline$H-2-821893$ & 4 & 2403WA & $\begin{array}{l}\text { Fire Protection Sprinkler System } \\
\text { Details and Sec }\end{array}$ \\
\hline$H-2-821893$ & 5 & 2403WA & $\begin{array}{l}\text { Fire Protection Sprinkler System } \\
\text { Details and Sec }\end{array}$ \\
\hline$H-2-821893$ & 6 & 2403WA & $\begin{array}{l}\text { Fire Protection SprinkTer System } \\
\text { Details and Sec }\end{array}$ \\
\hline $\mathrm{H}-2-821893$ & 7 & 2403WA & $\begin{array}{l}\text { Fire Protection Sprinkler System } \\
\text { Details and Sec }\end{array}$ \\
\hline$H-2-821893$ & 8 & 2403WA & $\begin{array}{l}\text { Fire Protection Sprinkler System } \\
\text { Details and Sec }\end{array}$ \\
\hline$H-2-80902$ & 1 & $2403 W B$ & $\begin{array}{l}\text { Fire Protection Alarm Sys Plan, } \\
\text { Sections }\end{array}$ \\
\hline $\mathrm{H}-2-80903$ & 1 & $2403 W B$ & Fire Protection Alarm Sys Wiring Diag \\
\hline $\mathrm{H}-2-80909$ & 1 & $2403 \mathrm{WB}$ & Instrumentation CAM Enclosure \\
\hline $\mathrm{H}-2-80909$ & 2 & $2403 W B$ & Instr CAM Enciosure \\
\hline $\mathrm{H}-2-80909$ & 3 & $2403 \mathrm{WB}$ & Instr CAM Enclosure \\
\hline $\mathrm{H}-2-80910$ & 1 & 2403WB & $\begin{array}{l}\text { Instrumentation Cam Alarm Inticon } \\
\text { Diag \& Instr Encl }\end{array}$ \\
\hline $\mathrm{H}-2-80910$ & 2 & $2403 W B$ & $\begin{array}{l}\text { Instrumentation Cam Alarm Elementary } \\
\text { Diagram }\end{array}$ \\
\hline $\mathrm{H}-2-80910$ & 3 & $2403 W B$ & $\begin{array}{l}\text { Instrumentation Flow Switch Alarm } \\
\text { Elementary Diagram }\end{array}$ \\
\hline $\mathrm{H}-2-80918$ & 1 & $2403 \mathrm{WB}$ & Electrical Plan \\
\hline $\mathrm{H}-2-80921$ & 1 & $2403 W B$ & Elec Elevations \& Details \\
\hline $\mathrm{H}-2-821894$ & 1 & $2403 W B$ & Fire Protection Sprinkler System \\
\hline$H-2-821894$ & 2 & 2403WB & $\begin{array}{l}\text { Fire Protection Sprinkler System } \\
\text { Details and Sec }\end{array}$ \\
\hline
\end{tabular}


HNF-SD-WM-TI-404, Rev. 5

Table 2. Support Drawings (cont.).

\begin{tabular}{|c|c|c|c|}
\hline $\begin{array}{l}\text { Drawing } \\
\text { No. }\end{array}$ & Sheet & Building & Title \\
\hline$H-2-821894$ & 3 & $2403 \mathrm{WB}$ & $\begin{array}{l}\text { Fire Protection Sprinkler System } \\
\text { Details and Sec }\end{array}$ \\
\hline$H-2-821894$ & 4 & $2403 W B$ & $\begin{array}{l}\text { Fire Protection Sprinkler System } \\
\text { Detajls and Sec }\end{array}$ \\
\hline$H-2-821894$ & 5 & $2403 W B$ & $\begin{array}{l}\text { Fire Protection Sprinkler System } \\
\text { Details and Sec }\end{array}$ \\
\hline $\mathrm{H}-2-821894$ & 6 & $2403 W B$ & $\begin{array}{l}\text { Fire Protection Sprinkler System } \\
\text { Details and Sec }\end{array}$ \\
\hline $\mathrm{H}-2-821894$ & 7 & $2403 W B$ & $\begin{array}{l}\text { Fire Protection Sprinkler System } \\
\text { Details and Sec }\end{array}$ \\
\hline$H-2-80902$ & 2 & $2403 W C$ & $\begin{array}{l}\text { Fire Protection Alarm Sys Plan, } \\
\text { Sections }\end{array}$ \\
\hline $\mathrm{H}-2-80903$ & 2 & $2403 W C$ & Fire Protection Alarm Sys Wiring Diag \\
\hline $\mathrm{H}-2-80907$ & 2 & $2403 W C$ & Instr CAM Enclosure \\
\hline $\mathrm{H}-2-80907$ & 3 & $2403 W C$ & Instr CAM Enclosure \\
\hline$H-2-80908$ & 1 & $2403 W C$ & $\begin{array}{l}\text { Instrumentation Cam Alarm Intcon } \\
\text { Diag. \& Instr. Encl }\end{array}$ \\
\hline$H-2-80908$ & 2 & $2403 W C$ & $\begin{array}{l}\text { Instrumentation Cam Alarm Elementary } \\
\text { Diagram }\end{array}$ \\
\hline $\mathrm{H}-2-80908$ & 3 & $2403 \mathrm{WC}$ & $\begin{array}{l}\text { Instrumentation Flow Switch Alarm } \\
\text { Elementary Diagram }\end{array}$ \\
\hline $\mathrm{H}-2-80919$ & 1 & $2403 \mathrm{WC}$ & Electrical Plan \\
\hline $\mathrm{H}-2-80922$ & 1 & $2403 W C$ & Elec Elevations \& Details \\
\hline $\mathrm{H}-2-821895$ & 1 & $2403 W C$ & Fire Protection Piping Layout \\
\hline $\mathrm{H}-2-821895$ & 2 & $2403 W C$ & Fire Protection Piping Detail \\
\hline $\mathrm{H}-2-821895$ & 3 & $2403 W C$ & Fire Protection Piping Detail \\
\hline $\mathrm{H}-2-821895$ & 4 & $2403 W C$ & Fire Protection Piping Detail \\
\hline $\mathrm{H}-2-821895$ & 5 & $2403 \mathrm{WC}$ & Fire Protection Piping Detail \\
\hline $\mathrm{H}-2-821895$ & 6 & $2403 W C$ & Fire Protection Piping Detail \\
\hline $\mathrm{H}-2-821895$ & 7 & $2403 W C$ & Fire Protection Piping Detail \\
\hline $\mathrm{H}-2-80902$ & 3 & $2403 W D$ & $\begin{array}{l}\text { Fire Protection Alarm Sys Plan Sect \& } \\
\text { Det }\end{array}$ \\
\hline $\mathrm{H}-2-80903$ & 3 & 2403WD & Fire Protection Alarm Sys Wiring Diag \\
\hline
\end{tabular}


HNF-SD-WM-TI-404, Rev. 5

Table 2. Support Drawings (cont.).

\begin{tabular}{|c|c|c|c|}
\hline $\begin{array}{l}\text { Drawing } \\
\text { No. }\end{array}$ & Sheet & Building & Title \\
\hline $\mathrm{H}-2-80905$ & 1 & $2403 W D$ & Instrumentation CAM Enclosure \\
\hline $\mathrm{H}-2-80905$ & 2 & $2403 W D$ & Instrumentation CAM Enclosure \\
\hline $\mathrm{H}-2-80905$ & 3 & $2403 W D$ & Instrumentation CAM Enclosure \\
\hline $\mathrm{H}-2-80906$ & I & $2403 W D$ & $\begin{array}{l}\text { Instrumentation Cam Alarm Intcon Diag } \\
\& \text { Instr Encl }\end{array}$ \\
\hline $\mathrm{H}-2-80906$ & 2 & $2403 W D$ & $\begin{array}{l}\text { Instrumentation Cam Alarm Elementary } \\
\text { Diagram }\end{array}$ \\
\hline $\mathrm{H}-2-80906$ & 3 & 2403WD & $\begin{array}{l}\text { Instrumentation Flow Switch Alarm } \\
\text { Elementary Diagram }\end{array}$ \\
\hline $\mathrm{H}-2-80907$ & 1 & $2403 W D$ & Instrumentation CAM Enclosure \\
\hline $\mathrm{H}-2-80917$ & 1 & $2403 W D$ & Electrical Plan \\
\hline $\mathrm{H}-2-80920$ & $I$ & $2403 W D$ & Elec Elevations \& Detajls \\
\hline $\mathrm{H}-2-821896$ & 1 & $2403 W D$ & Fire Protection Piping Layout \\
\hline $\mathrm{H}-2-821896$ & 2 & $2403 \mathrm{WD}$ & Fire Protection Piping Detail \\
\hline $\mathrm{H}-2-821896$ & 3 & 2403WD & Fire Protection Piping Detail \\
\hline $\mathrm{H}-2-821896$ & 4 & $2403 W D$ & Fire Protection Piping Detail \\
\hline $\mathrm{H}-2-821896$ & 5 & $2403 W D$ & Fire Protection Piping Detail \\
\hline $\mathrm{H}-2-821896$ & 6 & 2403WD & Fire Protection Piping Detail \\
\hline $\mathrm{H}-2-821896$ & 7 & 2403WD & Fire Protection Piping Detail \\
\hline $\mathrm{H}-2-821896$ & 8 & 2403WD & Fire Protection Piping Detail \\
\hline $\mathrm{H}-2-821896$ & 9 & $2403 \mathrm{WD}$ & Fire Protection Piping Detail \\
\hline $\mathrm{H}-2-823229$ & 1 & $2404 W$ & HVAC/Instm Floor Plan \& Notes \\
\hline $\mathrm{H}-2-823230$ & 1 & $2404 \mathrm{~W}$ & HVAC/Instm Details \& Schedules \\
\hline $\mathrm{H}-2-823231$ & 1 & $2404 W$ & $\begin{array}{l}\text { Fire Protection Floor Plans Sect Det } \\
\text { \& Notes }\end{array}$ \\
\hline $\mathrm{H}-2-823236$ & 1 & $2404 W$ & Fire Protection Fire Alarm Plan \& Det \\
\hline$H-2-823237$ & 1 & $2404 W$ & $\begin{array}{l}\text { Fire Protection Fire Alarm Wiring } \\
\text { Details }\end{array}$ \\
\hline $\mathrm{H}-2-34081$ & 1 & $2727 W$ & Sodium Tank Pressure Monitoring \\
\hline $\mathrm{H}-2-34081$ & 2 & $2727 W$ & Sodium Tank Pressure Monitoring \\
\hline
\end{tabular}


HNF-SD-WM-TI-404, Rev. 5

Table 2. Support Drawings (cont.).

\begin{tabular}{||c|c|c|l||}
\hline $\begin{array}{c}\text { Drawing } \\
\text { No. }\end{array}$ & Sheet & Building & \multicolumn{1}{|c||}{ Title } \\
\hline $\mathrm{H}-2-34081$ & 3 & $2727 \mathrm{~W}$ & $\begin{array}{l}\text { Sodium Tank Pressure Monitoring } \\
\text { Connection Diagram }\end{array}$ \\
\hline $\mathrm{H}-2-34081$ & 4 & $2727 \mathrm{~W}$ & $\begin{array}{l}\text { Sodium Tank Pressure Monitoring } \\
\text { Connection Diagram }\end{array}$ \\
\hline $\mathrm{H}-2-34084$ & 1 & $2727 \mathrm{~W}$ & $\begin{array}{l}\text { Sodium Tank Stge Building Plans \& } \\
\text { Details }\end{array}$ \\
\hline $\mathrm{H}-2-34084$ & 2 & $2727 \mathrm{~W}$ & $\begin{array}{l}\text { Sodium Tank Stge Building Plans \& } \\
\text { Details }\end{array}$ \\
\hline $\mathrm{H}-2-97468$ & 1 & $2727 \mathrm{~W}$ & $\begin{array}{l}\text { Electrical Radio Fire Alarm Plan \& } \\
\text { Diagram }\end{array}$ \\
\hline $\mathrm{H}-2-72499$ & 1 & $2727 \mathrm{~W}$ & Sodium Storage Building \\
\hline $\mathrm{H}-2-72499$ & 2 & $2727 \mathrm{~W}$ & Sodium Storage Building \\
\hline
\end{tabular}

